\title{
Down-regulation of cellular FLICE-inhibitory protein (Long Form) contributes to apoptosis induced by Hsp90 inhibition in human lung cancer cells
}

Qilin Wang ${ }^{1,2+}$, Wendong Sun ${ }^{3+}$, Xuexi Hao ${ }^{3}$, Tianliang $\mathrm{Li}^{1}$, Ling Su${ }^{1 *}$ and Xiangguo $\mathrm{Liu}^{1,4^{*}}$

\begin{abstract}
Background: Cellular FLICE-Inhibitory Protein (long form, c-FLIPL) is a critical negative regulator of death receptor-mediated apoptosis. Overexpression of $\mathrm{c}-\mathrm{FLIP}_{\mathrm{L}}$ has been reported in many cancer cell lines and is associated with chemoresistance. In contrast, down-regulation of c-FLIP may drive cancer cells into cellular apoptosis. This study aims to demonstrate that inhibition of the heat shock protein 90 ( $\mathrm{Hsp} 90$ ) either by inhibitors geldanamycin/17-N-Allylamino-17-demethoxygeldanamycin (GA/17-AAG) or siRNA technique in human lung cancer cells induces C-FLIP degradation and cellular apoptosis through C-terminus of Hsp70-interacting protein (CHIP)-mediated mechanisms.
\end{abstract}

Methods: Calu-1 and H157 cell lines (including H157-c-FLIPL overexpressing C-FLIPL and control cell H157-lacZ) were treated with 17-AAG and the cell lysates were prepared to detect the given proteins by Western Blot and the cell survival was assayed by SRB assay. CHIP and Hsp90 a/ $\beta$ proteins were knocked down by siRNA technique. CHIP and $c-F L I P_{L}$ plasmids were transfected into cells and immunoprecipitation experiments were performed to testify the interactions between c-FLIP ${ }_{L}$ CHIP and Hsp90.

Results: C-FLIP down-regulation induced by 17-AAG can be reversed with the proteasome inhibitor MG132, which suggested that c-FLIP degradation is mediated by a ubiquitin-proteasome system. Inhibition of Hsp90a/ $\beta$ reduced c-FLIPL level, whereas knocking down CHIP expression with siRNA technique inhibited c-FLIPL degradation.

Furthermore, $c-F L I P_{L}$ and CHIP were co-precipitated in the IP complexes. In addition, overexpression of c-FLIP $P_{L}$ can rescue cancer cells from apoptosis. When 17-AAG was combined with an anti-cancer agent celecoxib(CCB), c-FLIPL level declined further and there was a higher degree of caspase activation.

Conclusion: We have elucidated c-FLIPL degradation contributes to apoptosis induced by Hsp90 inhibition, suggesting C-FLIP and Hsp90 may be the promising combined targets in human lung cancer treatment.

Keywords: c-FLIPL, Apoptosis, CHIP, Hsp90

\footnotetext{
* Correspondence: suling@sdu.edu.cn; xgliu@sdu.edu.cn

${ }^{\dagger}$ Equal contributors

'Key Laboratory for Experimental Teratology of the Ministry of Education and

School of Life Sciences, Shandong University, Jinan, China

${ }^{4}$ Shandong University School of Life Sciences, Room103, South Building, 27

Shandananlu Road, Jinan 250100, China

Full list of author information is available at the end of the article
} 


\section{Introduction}

Caspase-8 activation plays an important role in the death receptor-mediated extrinsic apoptotic pathway in human cancer cells [1]. When binding to ligands, the death receptor is activated and forms the death induced signal complex (DISC) together with FADD and procaspase- 8 . Procaspase- 8 is activated and initiates the caspase cascade which mediates cellular apoptosis. c-FLIP ${ }_{\mathrm{L}}$ is a major protein that can prevent caspase-8/10 activation in the DISC and inhibiting apoptosis mediated by death receptors. It has been found that c-FLIP ${ }_{\mathrm{L}}$ is upregulated in several carcinomas $[2,3]$ and overexpression of $\mathrm{c}$-FLIP $\mathrm{L}$ can be responsible for chemoresistance and malignant transformation [4-6]. To date, more than 10 splicing variants of c-FLIP genes have been identified at the mRNA level. However, only c-FLIP $\mathrm{L}_{\mathrm{L}}$ and $\mathrm{c}$-FLIP have been extensively studied at the protein level. Although these two splicing variants have distinct structural and functional properties, they have been found to be recruited to DISC and to inhibit caspase- 8 activation. Recent studies have shown that c-FLIP ${ }_{\mathrm{L}}$ degradation is dependent on JNK-mediated E3-ligase Itch and AKT phosphorylation, while degradation of c-FLIP ${ }_{S}$ seems to have different pattern $[7,8]$.

Hsp90 is a pleiotropic molecular chaperone and functions as a key protein in the conformational maturation and stability of client proteins, many of which are kinases, cell cycle regulators and steroid receptors, etc. [9]. These client proteins play important roles in signaling transduction, cell proliferation and cancer chemoresistance. The Hsp90based molecular chaperone complex interacts with its client proteins in an iterative way. Hsp90 cycles are driven by ATP- or ADP-bound conformations that are mediated via multiple rounds of ATP binding and hydrolysis [9]. Hsp90 inhibitors such as geldanamycin (GA) or its synthetic analogue 17-AAG, directly binds to the ATP-binding pocket in the N-terminal domain of Hsp90 and, hence, blocks the binding of nucleotides to Hsp90. Once Hsp90 activity is inhibited, the Hsp90-dependent protein dissociates from the multi-chaperone complexes and is targeted for degradation by the ubiquitin-proteasome system [10,11]. 17-AAG-induced apoptosis has been previously reported to be associated with $\mathrm{c}-\mathrm{FLIP}_{\mathrm{S}}$ down-regulation [12]. However, the relationship between Hsp90 and c-FLIP ${ }_{\mathrm{L}}$ has not been well elucidated.

C-terminus of Hsp70-interacting protein (CHIP) has the E3 ligase activity, and it binds to Hsp/Hsc70 and Hsp90 complex by means of its TPR (an amino-terminal tetratricopeptide) domain [13]. It has been reported that CHIP degrades Hsp90 client proteins, such as the glucocorticoid receptor, the cystic fibrosis transmembrane-conductance regulator and ErbB2 [13-15]. In this study, we show that CHIP is involved in c-FLIP ${ }_{\mathrm{L}}$ degradation induced by Hsp90 inhibition. Since both Hsp90 and c-FLIP ${ }_{L}$ play a critical role in oncogenesis and chemoresistance, inhibition of Hsp90 by 17-AAG in combination with therapeutic agents targeting c-FLIP may be an efficacious strategy in patients with tumors that express high levels of c-FLIP ${ }_{\mathrm{L}}$ and other Hsp90 client proteins.

\section{Results}

Hsp90 inhibitor 17-AAG and GA induces C-FLIP ${ }_{L}$ downregulation via ubiquitin-proteasome pathway in NSCLC cells

17-AAG-induced apoptosis has been previously reported to be associated with c-FLIP ${ }_{S}$ down-regulation [12]. However the relationship between 17-AAG/GA and c-FLIP ${ }_{L}$ has not been well elucidated. In this study we examined the effect of c-FLIP ${ }_{L}$ expression induced by 17-AAG or GA in lung cancer cell lines including Calu-1, A549, H460 and H157. After treatment with 17-AAG and GA at the indicated concentrations for $48 \mathrm{~h}, \mathrm{c}-\mathrm{FLIP}_{\mathrm{L}}$ levels were reduced (Figure 1A and $\mathrm{B}$ ). We also performed time course experiments to monitor the expression of $\mathrm{C}-\mathrm{FLIP}_{\mathrm{L}}$. Calu-1 and $\mathrm{H} 157$ cells were treated with 17-AAG $(1.0 \mu \mathrm{M})$ or $\mathrm{GA}(4.0 \mu \mathrm{M})$ for the indicated time. We found that the c-FLIP ${ }_{\mathrm{L}}$ expression decreased in a time-dependent manner. In Calu-1 and H157 cells, the time of c-FLIP ${ }_{\mathrm{L}}$ downregulation occurred as early as $4 \mathrm{~h}$ and was sustained for at least $48 \mathrm{~h}$ following 17-AAG or GA exposure (Figure 1C).

It has been reported that c-FLIP $\mathrm{L}_{\mathrm{L}}$ degradation is correlated with ubiquitin [7]. To determine whether 17-AAG induces proteasome-mediated c-FLIP ${ }_{\mathrm{L}}$ degradation, we tested the effects of 17-AAG and GA on c-FLIP $\mathrm{L}_{\mathrm{L}}$ expression in the absence or presence of the proteasome inhibitor MG132 in Calu-1 and H157 cells. As shown in Figure 2A and B, MG132 at the concentration of $20 \mu \mathrm{M}$ abrogated the ability of 17-AAG and GA to reduce c-FLIP $_{\mathrm{L}}$, suggesting that 17-AAG- and GA-induced c-FLIP $\mathrm{L}_{\mathrm{L}}$ down-regulation is dependent on proteasomemediated degradation pathway.

\section{Inhibition of Hsp90 expression induces C-FLIP $\mathrm{L}_{\mathrm{L}}$ down- regulation}

It has been documented that 17-AAG and GA induced degradation of Hsp90 client proteins [9]. Since we detected that $\mathrm{c}-\mathrm{FLIP}_{\mathrm{L}}$ down-regulation was induced by 17-AAG and GA, we wondered whether c-FLIP $\mathrm{L}_{\mathrm{L}}$ was a client protein of Hsp90. We knocked down Hsp90 $\alpha / \beta$ with siRNA and then detected the c-FLIP ${ }_{L}$ and $\mathrm{Hsp} 90 \alpha / \beta$ protein levels by Western blot. Hsp $90 \alpha / \beta$ protein levels were reduced after knockdown, and the c-FLIP ${ }_{\mathrm{L}}$ level was also decreased compared to the control (Figure 3A and B), suggesting Hsp90 controls the stability of c-FLIP $\mathrm{L}_{\mathrm{L}}$ protein. 


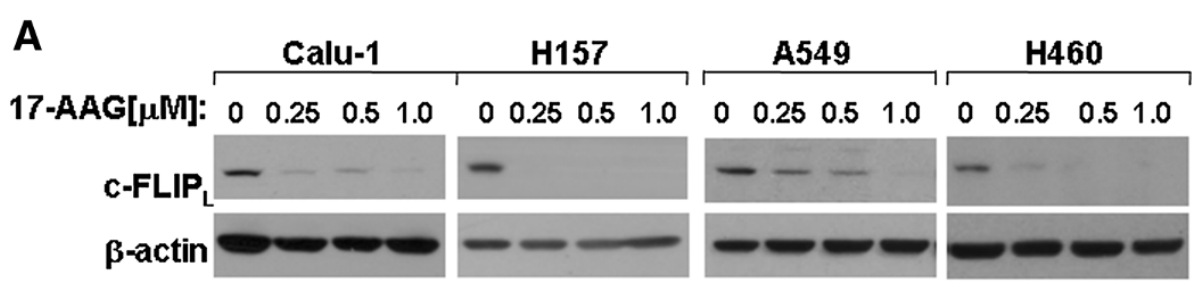

B

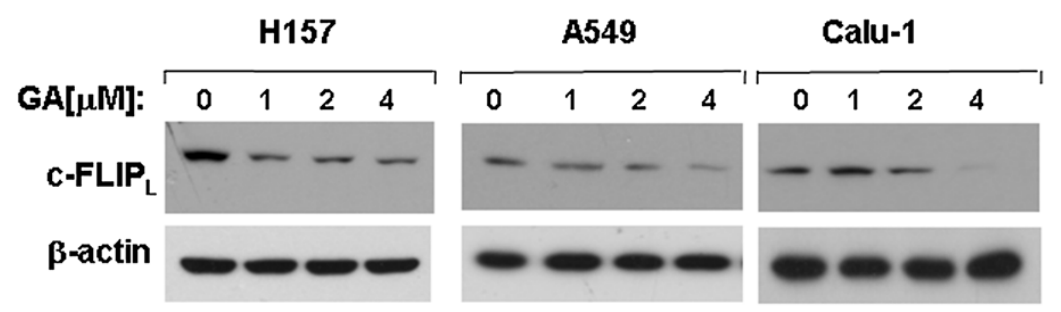

C

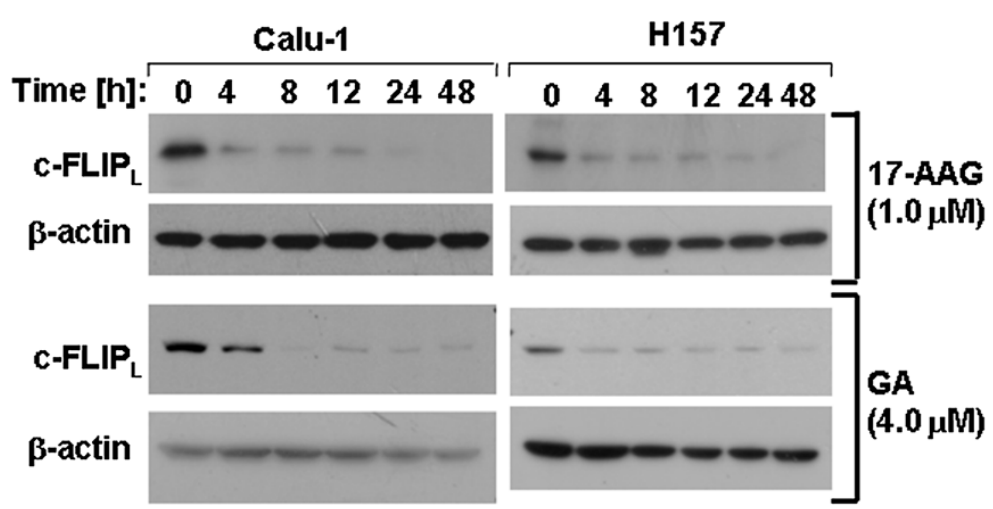

Figure 1 17-AAG and GA induces C-FLIP down-regulation in NSCLC cells. (A) and (B), Calu-1, H157, A549 and H460 cell lines were treated

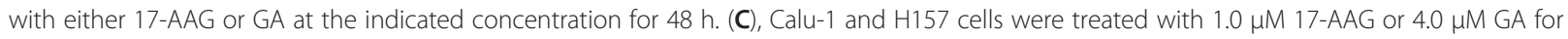
the indicated time. The cells were subjected to preparation of whole-cell protein lysates and C-FLIPL proteins were detected by Western blot analysis.

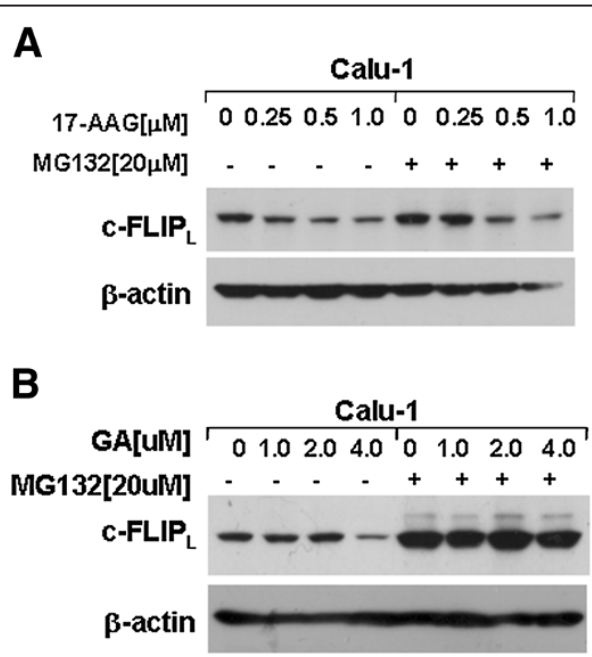

Figure 2 17-AAG and GA induce c-FLIP $\mathrm{L}_{\mathrm{L}}$ down-regulation via proteasome pathway. Calu-1 and $\mathrm{H} 157$ cells were pretreated with $20 \mu \mathrm{M}$ MG132 for 30 min and co-treated with the indicated concentrations of 17-AAG (A) or GA (B) for $4 \mathrm{~h}$, and then the cells were subjected to preparation of whole-cell protein lysates and C-FLIPL proteins were detected by Western blot analysis. 


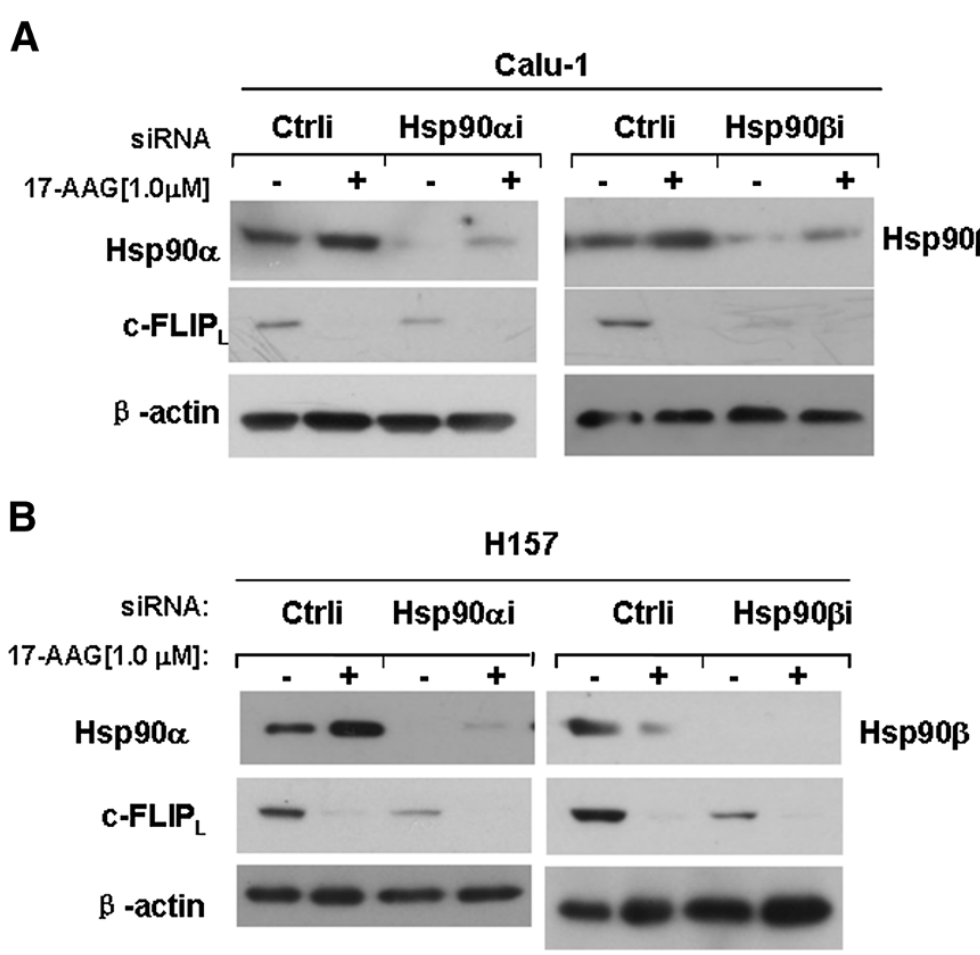

Figure 3 Inhibition of Hsp90 expression facilitates c-FLIP $\mathbf{L}_{\mathbf{L}}$ down-regulation. (A) and (B), Calu-1 and H157 cells were transfected with siRNAs of control, Hsp90a and Hap90ß, respectively. Twenty four hours later, cells were treated with 1.0 $\mu \mathrm{M}$ 17-AAG for $48 \mathrm{~h}$. The cells were harvested for Western blot analysis to detect Hsp90a/ $\beta$ and c-FLIP levels.

CHIP modulates the C-FLIP $\mathrm{L}_{\mathrm{L}}$ degradation induced by 17-AAG CHIP interacts with Hsp90 chaperone complexes and displays E3-ligase activity to degrade Hsp90 client proteins $[13,15]$. In order to detect the interaction of CHIP with c-FLIP ${ }_{\mathrm{L}}$, we explored dose experiments in

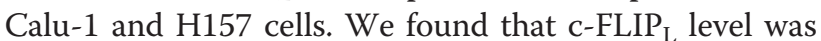
reduced while CHIP expression was slightly increased after treatment with 17-AAG (Figure 4A and B). Furthermore, when CHIP was knocked down, the degradation of c-FLIP ${ }_{\mathrm{L}}$ was abrogated (Figure 4C) in Calu-1 and H157 cells, which indicated that $\mathrm{c}-\mathrm{FLIP}_{\mathrm{L}}$ degradation was modulated by CHIP.

\section{CHIP interacts with $\mathrm{C}-\mathrm{FLIP}_{\mathrm{L}}$ in vivo and promotes the} ubiquitination of $\mathrm{C}-\mathrm{FLIP}_{\mathrm{L}}$

To further test if there is physically interaction between CHIP and $c-F_{L} I_{L}$, immunoprecipitation experiments were performed. Calu-1 cells were transfected with plasmids carrying Myc-tagged CHIP or Flag-tagged c-FLIP $\mathrm{L}_{\mathrm{L}}$ and then treated with $1.0 \mu \mathrm{M}$ 17-AAG for $4 \mathrm{~h}$, the lysate supernatant was incubated with Myc antibody for $1 \mathrm{~h}$ and then with protein-G and protein-A (1:1 mix) beads overnight (left panel), or anti-FLAG M2 Affinity Gel beads overnight directly(right panel). Western Blot analysis showed that

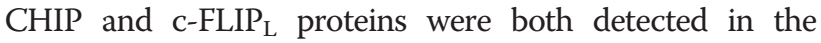
immunoprecipitation complexes, suggesting that both exogenous and endogenous CHIP interacts with c-FLIP physically in vivo. Hsp90 was also pulled down together with $\mathrm{C}-\mathrm{FLIP}_{\mathrm{L}}$ and CHIP, suggesting that CHIP interacts with c-FLIP $\mathrm{L}_{\mathrm{L}}$ in an Hsp90 chaperone complex (Figure 4D). Considering CHIP can be an E3 ligase, we wonder if it can regulate the ubiquitination level of $\mathrm{c}-\mathrm{FLIP}_{\mathrm{L}}$. H157-c-FLIPL cells were transfected with plasmids carrying Myc-tagged CHIP or Myc-tagged CHIP (H260Q Mutant) and then treated with $1.0 \mu \mathrm{M}$ 17-AAG for $8 \mathrm{~h}$. After immunoprecipitaion with Flag antibody, Flag-tagged $\mathrm{c}-\mathrm{FLIP}_{\mathrm{L}}$ was pulled down. Western Blot analysis showed that CHIP overexpression promoted the $\mathrm{c}-\mathrm{FLIP}_{\mathrm{L}}$ ubiquitination, while the mutant CHIP overexpression suppressed the $\mathrm{c}^{-\mathrm{FLIP}_{\mathrm{L}}}$ ubiquitination after 17-AAG treatment (Figure 4E). Taken together, our data suggest that CHIP interacts with $\mathrm{C}-\mathrm{FLIP}_{\mathrm{L}}$ in vivo and promotes the ubiquitination of $\mathrm{c}-\mathrm{FLIP}_{\mathrm{L}}$.

\section{Ectopic overexpression of C-FLIP L protects cancer cells from apoptosis}

To determine the effect of $\mathrm{c}-\mathrm{FLIP}_{\mathrm{L}}$ overexpression on 17-AAG- and GA-induced apoptosis, two cell clones H157-C-FLIP $_{\mathrm{L}}$ and H157-LacZ that overexpress C-FLIP and $L a c Z$ respectively were used to examine their effects on 17-AAG- and GA-induced caspase activation and apoptosis. By Western blotting, we detected high levels of ectopic c-FLIP $\mathrm{L}_{\mathrm{L}}$ in the H157-c-FLIP $\mathrm{L}_{\mathrm{L}}$ cell line. We 
A

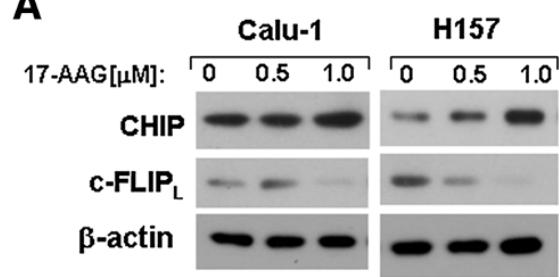

B

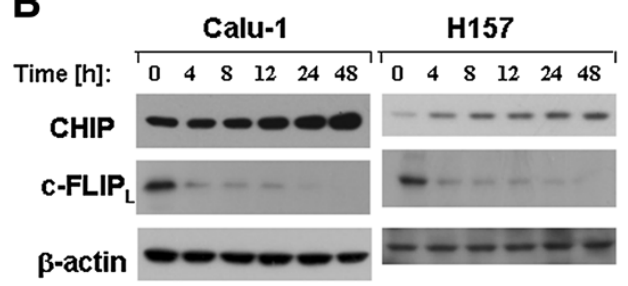

C

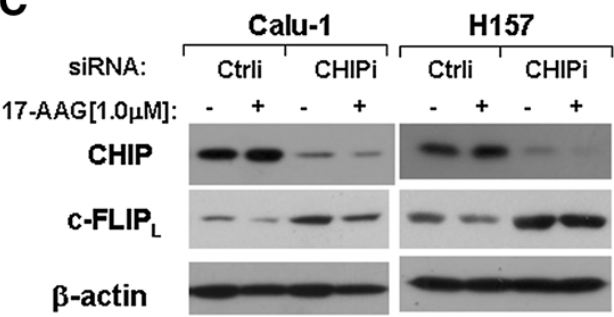

D

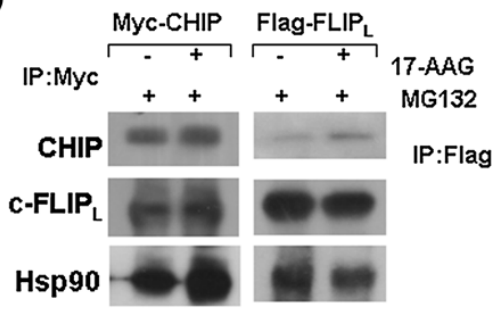

E

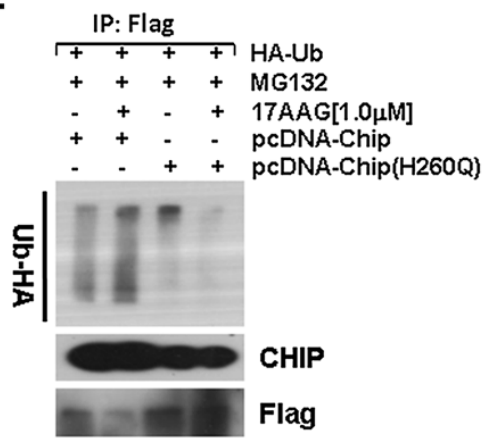

Figure 4 CHIP is involved in C-FLIP degradation. (A), Calu-1 and H157 cells were treated with the indicated concentrations of 17-AAG for 48 h. (B), Calu-1 and H157 cells were treated with $1.0 \mu \mathrm{M}$ 17-AAG for the indicated time. (C), Calu-1 and H157 cells were transfected with control siRNA (Crtli) and CHIP siRNA (CHIPi). $24 \mathrm{~h}$ after transfection, cells were reseeded and treated with $1.0 \mu \mathrm{M}$ 17-AAG for $48 \mathrm{~h}$. (D), Calu-1 cells were transfected with pcDNA-Myc-CHIP plasmid, and the cells were treated with $20 \mu \mathrm{M} \mathrm{MG132}$ and $1.0 \mu \mathrm{M}$ 17-AAG for 4 h. For immunoprecipitation, cell lysate was incubated with Myc antibody for $1 \mathrm{~h}$ and then incubated with protein A/G agarose $(1: 1 \mathrm{mix})$ at $4^{\circ} \mathrm{C}$ overnight(Left panel). Calu-1 cells were transfected with Lenti-Flag-c-FLIPL, and the cells were treated with $1.0 \mu \mathrm{M}$ 17-AAG and $20 \mu \mathrm{M}$ MG132 for 4 h. For immunoprecipitation, cell lysate was incubated with Anti-FLAG M2 beads at $4^{\circ} \mathrm{C}$ overnight (Right panel). The precipitated proteins were analyzed by Western Blot assay. (E), H157/c-FLIP cells were transfected with plasmids Ub-HA and pcDNA-Myc-CHIP or pcDNA-Myc-CHIP(H260Q mutant), and then the cells were treated with 1.0 $\mu \mathrm{M}$ 17-AAG and $20 \mu \mathrm{M}$ MG132 for $8 \mathrm{~h}$. The cell lysates were subjected to immunoprecipitation with Flag antibody and subsequently Western Blot analysis.

found that 17-AAG or GA induced activation of caspase-8, caspase-9, caspase-3 and cleavage of PARP in H157-LacZ control cells, as demonstrated by increased levels of cleaved bands (Figure 5A). This was not seen in the corresponding H157-c-FLIP $\mathrm{L}_{\mathrm{L}}$ cells. We measured the survival of H157-LacZ to be $32.4 \%$ in cells treated with $2.0 \mu \mathrm{M}$ 17-AAG for $48 \mathrm{~h}$, whereas the survival of H157-c-FLIP ${ }_{L}$ cells were more than 58.1\% (Figure 5B) by SRB assay. The similar result was observed for SRB assay in GA-treated cells. From the above results, we conclude that ectopic overexpression of $\mathrm{c}-\mathrm{FLIP}_{\mathrm{L}}$ protects cancer cells from apoptosis induced by Hsp90 inhibition.

\section{7-AAG enhances celecoxib-induced c-FLIP $\mathrm{P}_{\mathrm{L}}$ down- regulation and apoptosis}

We have reported that celecoxib (CCB) down-regulates c-FLIP ${ }_{\mathrm{L}}$ through ubiquitin-proteasome degradation [16]. To determine whether 17-AAG enhances celecoxib-

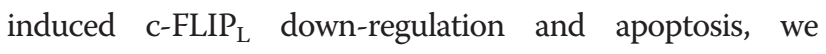

investigated the level of $c$-FLIP $\mathrm{L}_{\mathrm{L}}$ expression and caspase activation by combination treatment with $\mathrm{CCB}$ and 17-AAG in Calu-1 cells. We found that 17-AAG combination with CCB had synergistic effects on apoptosis induction by Western blotting and SRB assay (Figure 6A and B). c-FLIP $\mathrm{L}_{\mathrm{L}}$ expression decreased further when 17-AAG was combined with $\mathrm{CCB}$ compared with the monotherapy Calu-1 cells. In addition, the activation of caspase-8, caspase-9, caspase- 3 and the cleavage of PARP were more pronounced. This data suggests that 17-AAG may enhance chemotherapeutic agent-induced $\mathrm{c}-\mathrm{FLIP}_{\mathrm{L}}$ down-regulation and apoptosis in lung cancer cells.

\section{Discussion}

Hsp90 plays a key role in conformational maturation and stability of client proteins in transducing proliferative and anti-apoptotic signals [10]. In the present study we found that Hsp90 inhibitor 17-AAG and GA or Hsp90 knockdown induced c-FLIP ${ }_{\mathrm{L}}$ down-regulation 

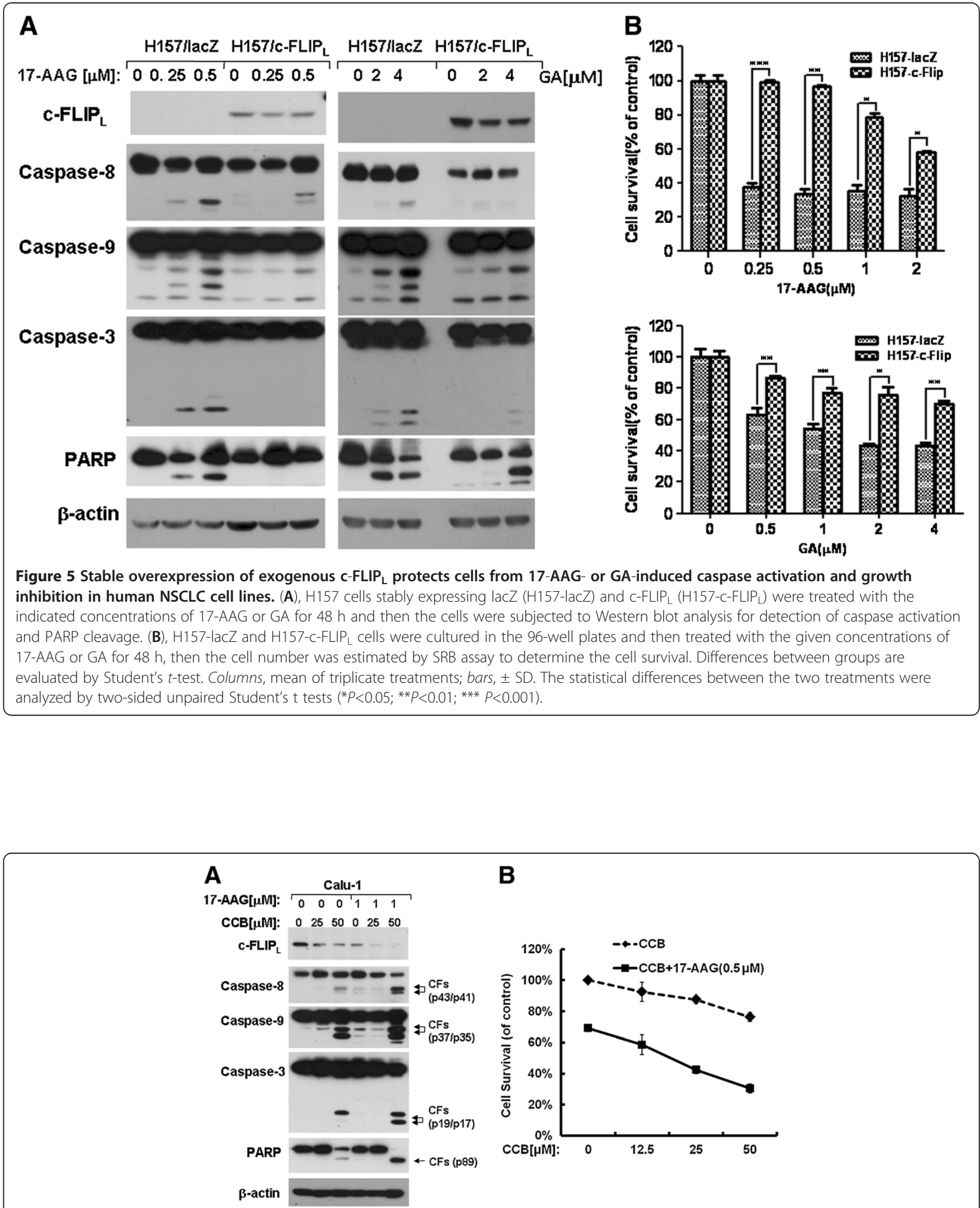

B

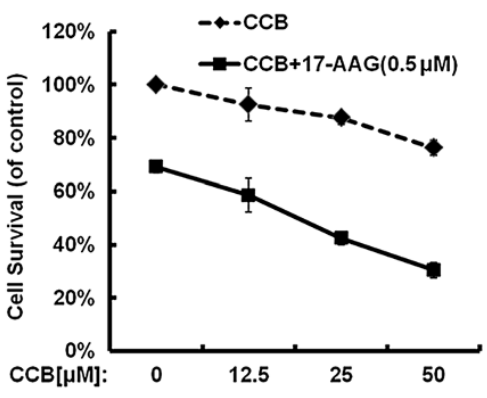

Figure 6 17-AAG enhances celecoxib-induced c-FLIP $\mathbf{L}_{\mathbf{L}}$ down-regulation and apoptosis. (A), Calu-1 cells were treated with 17-AAG, celecoxib alone, and their respective combinations. After treatment for $48 \mathrm{~h}$, the cells were subjected to detection of caspase activation and PARP by Western blot assay. (B), Calu-1 cells were seeded in 96-well plate and on the second day were treated with the given concentrations of CCB or combined concentrations of 17-AAG and CCB for $48 \mathrm{~h}$, and then the cell number was estimated by SRB assay to determine the cell survival. 
which subsequently resulted in cancer cell apoptosis. Although DR5 is an important death receptor in the extrinsic apoptotic pathway, in the above experiments, we failed to detect DR5 up-regulation (data no shown). This indicates that $\mathrm{c}-\mathrm{FLIP}_{\mathrm{L}}$ may primarily contribute to Hsp90 inhibition-induced apoptosis in lung cancer cells.

Currently, more than one hundred client proteins of Hsp90 have been identified, and they are degraded when Hsp90 multi-chaperone complex activity is disrupted by the inhibitors. When we inhibited Hsp90 expression using siRNA technique and examined the c-FLIP ${ }_{\mathrm{L}}$ level, we found c-FLIP ${ }_{L}$ was reduced in the cells whose Hsp90 was knocked down, suggesting that $\mathrm{c}-\mathrm{FLIP}_{\mathrm{L}}$ is a client protein of Hsp90. It is reported when Hsp90 is inhibited, it recruits E3 ligase (e.g. CHIP) to degrade the client proteins [13-15]. When we knocked down CHIP, c-FLIP ${ }_{L}$ degradation was inhibited after treatment with 17-AAG, which indicated that CHIP modulated $\mathrm{c}-\mathrm{FLIP}_{\mathrm{L}}$ degradation in the NSCLC cell lines. Next, we performed immunoprecipitation experiments in Calu-1 cell lines. We found that CHIP was co-precipitated with c-FLIP in the precipitated complex. We also found that Hsp90 was precipitated at the same time, hinting that $\mathrm{c}-\mathrm{FLIP}_{\mathrm{L}}$ is the client protein of Hsp90 and CHIP modulates

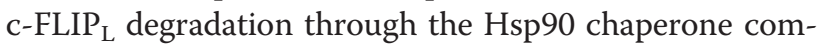
plex. Itch is reported as an E3 ligase of $c-\mathrm{FLIP}_{\mathrm{L}}$ in the mouse cells upon exposure to TNFa [7]. However, it has also been reported that some agents induced c-FLIP degradation independently of Itch [17-19]. Additionally, we found that CHIP promotes the ubiquitination of c-FLIP, indicating CHIP regulates c-FLIP $_{\mathrm{L}}$ degradation through uibiquitination when the cells were treated with 17-AAG.

It has been investigated that the clinically used anti-

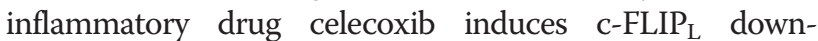
regulation and facilitates cancer cell apoptosis alone or in combination with other agents [16]. We found that 17-AAG and celecoxib had synergistic effects in promoting c-FLIP $\mathrm{L}_{\mathrm{L}}$ down-regulation and cellular apoptosis. In addition, overexpression of $\mathrm{c}-\mathrm{FLIP}_{\mathrm{L}}$ could rescue cancer cells from apoptosis. These data suggest that $\mathrm{c}-\mathrm{FLIP}_{\mathrm{L}}$ down-regulation plays important role in Hsp90 inhibition-induced apoptosis in NSCLC cells.

\section{Conclusions}

Our data reveals that $\mathrm{c}-\mathrm{FLIP}_{\mathrm{L}}$ is down-regulated and apoptosis is induced effectively by Hsp90 inhibition, either by 17AAG and GA or siRNA technique, and $\mathrm{c}-\mathrm{FLIP}_{\mathrm{L}}$ is degraded via proteasome-mediated pathway. CHIP modulates c-FLIP degradation induced by $\mathrm{Hsp} 90$ inhibition in human NSCLC cell lines. Targeting both c-FLIP ${ }_{L}$ and Hsp90 may represent a promising therapeutic strategy for the patients whose tumors overexpress c-FLIP $\mathrm{L}_{\mathrm{L}}$ and Hsp90 client proteins.

\section{Materials and methods Reagents and antibodies}

17-AAG and GA were purchased from LC Laboratories (Pompano Beach). Celecoxib, MG132 and Anti-FLAG M2 Affinity Gel were purchased from Sigma (St. Louis, MO). Protein A and Protein G Agarose were purchased from Roche (Mannheim, Germany).

Mouse anti-caspase- 3 and anti-caspase- 8 monoclonal antibodies, rabbit anti-caspase- 9 and anti-poly (ADP-ribose) polymerase (PARP) antibodies were purchased from Cell Signaling Technology (Danvers, MA). Mouse anti-FLIP ${ }_{L}$ monoclonal antibody was purchased from Alexis Biochemicals (San Diego, CA). Mouse anti-Hsp90 antibodies were purchased from Abcam (Cambridge, UK). Mouse anti-Myc and anti$\beta$-actin antibodies, rabbit anti-Flag and anti-CHIP antibodies were purchased from Sigma (St. Louis, MO).

\section{Cell lines, cell culture and transfection}

The human non-small cell lung cancer cell lines used in this study were obtained from the American Type Culture Collection (Manassas, VA) and were grown in RPMI 1640 supplemented with $5 \%$ fetal bovine serum at $37^{\circ} \mathrm{C}$ in a humidified atmosphere of $5 \% \mathrm{CO}_{2}$ and $95 \%$ air. Cells were transfected with different plasmids by using the FuGene reagent from Roche (Indianapolis, IN), according to the manufacturer's instructions.

\section{Cell survival assay}

Cells were seeded in 96-well plate with an appropriate amount in $100 \mu \mathrm{L}$ culture medium per well. On the second day, compounds or drugs were mixed in and the total volume of per well was $200 \mu \mathrm{L}$. Cells were treated for defined time and then cell viability was measured by sulforhodamine B (SRB) assay as described previously [20].

\section{Western blot analysis}

The whole-cell protein lysates preparation and Western blot analysis were described in reference [21].

\section{Plasmids and establishment of stable cell lines that overexpress c-FLIP}

Plasmids of Myc-CHIP and Myc-CHIP (H260Q) were kindly provided by Dr. Hamid Band (University of Nebraska Medical Center, USA). Lenti-Flag-c-FLIP L was constructed before [22]. The cell clones that can overexpress c-FLIP $\mathrm{L}_{\mathrm{L}}$ and $\mathrm{LacZ}$ were constructed as previously described [22].

\section{Knockdown of Hsp90, CHIP expression with siRNA}

siRNAs were synthesized by GenePharma (Shanghai, China). Control siRNA and Hsp90 $\alpha / \beta$ siRNA target 
sequences were described before [23]. The target sequence of CHIP siRNA was 5'-AGGCCAAGCACGACAAGTA- $3^{\prime}$. Transfection of siRNA was conducted as previously described [22].

\section{Immunoprecipitation}

To precipitate Flag-tagged FLIP $_{\mathrm{L}}$ or Myc-tagged CHIP proteins, cells were lysed with lysis buffer and Anti-FLAG M2 Affinity Gel beads or Myc antibody with Protein A/G Agarose beads (1:1 mix) were used to pull down Flag-tagged $\mathrm{FLIP}_{\mathrm{L}}$ or Myc-tagged CHIP proteins according to the manufacture's instructions.

\section{Competing interests}

The authors declare that they have no competing interests.

\section{Authors' contributions}

XGL and LS conducted the project design. QLW, WDS, XXH, TLL and LS conducted the experiments and data analysis. QL W and XG $\mathrm{L}$ drafted the manuscript. XG $L$ managed the funding acquisition, supervised the project and was involved in interpretation of data and revision of the manuscript. All authors have contributed and approved the final manuscript.

\section{Acknowledgements}

We thank Dr. Hamid Band (University of Nebraska Medical Center, USA) for kindly providing the plasmids of Myc-CHIP and Myc-CHIP (H260Q). We thank Dr. Sunil Singhal for English editing.

This work was supported by grants from the National Natural Science Foundation of China (31071215 and 30971307), National Science Major Project from Ministry of Science and Technology of China (2013CB910900 project 3) and Shandong Provincial Natural Science Foundation (2010GSF10218 and JQ201007).

\section{Author details}

${ }^{1}$ Key Laboratory for Experimental Teratology of the Ministry of Education and School of Life Sciences, Shandong University, Jinan, China. ${ }^{2}$ Liaocheng University School of Life Sciences, Liaocheng, China. ${ }^{3}$ The Second Hospital, Shandong University, Jinan, China. ${ }^{4}$ Shandong University School of Life Sciences, Room103, South Building, 27 Shandananlu Road, Jinan 250100, China.

Received: 26 November 2012 Accepted: 20 December 2012

Published: 21 December 2012

\section{References}

1. Wang F, Du L, Wang X: TNF-alpha induces two distinct caspase-8 activation pathways. Cell 2008, 133:693-703.

2. Salon C, Eymin B, Micheau O, Chaperot L, Plumas J, Brambilla C, Brambilla E, Gazzeri S: E2F1 induces apoptosis and sensitizes human lung adenocarcinoma cells to death-receptor-mediated apoptosis through specific downregulation of c-FLIP(short). Cell Death Differ 2006, 13:260-272.

3. Zong H, Yin B, Chen J, Ma B, Cai D, He X: Over-expression of c-FLIP confers the resistance to TRAIL-induced apoptosis on gallbladder carcinoma, Tohoku. J Exp Med 2009, 217:203-208.

4. Wilson TR, McLaughlin KM, McEwan M, Sakai H, Rogers KM, Redmond KM, Johnston PG, Longley DB: c-FLIP: a key regulator of colorectal cancer cell death. Cancer Res 2007, 67:5754-5762.

5. Abedini MR, Qiu Q, Yan X, Tsang BK: Possible role of FLICE-like inhibitory protein (FLIP) in chemoresistant ovarian cancer cells in vitro. Oncogene 2004, 23:6997-7004.

6. Duiker EW, van der Zee AG, de Graeff P, Boersma-van EW, Hollema H, de Bock $\mathrm{GH}$, de Jong S, de Vries E: The extrinsic apoptosis pathway and its prognostic impact in ovarian cancer. Gynecol Oncol 2010, 116:549-555.

7. Chang L, Kamata H, Solinas G, Luo JL, Maeda S, Venuprasad K, Liu YC, Karin M: The E3 ubiquitin ligase itch couples JNK activation to TNFalphainduced cell death by inducing c-FLIP(L) turnover. Cell 2006, 124:601-613.
8. Shi B, Tran T, Sobkoviak R, Pope RM: Activation-induced degradation of FLIP $(L)$ is mediated via the phosphatidylinositol 3-kinase/Akt signaling pathway in macrophages. J Biol Chem 2009, 284:14513-14523.

9. Kamal A, Boehm MF, Burrows FJ: Therapeutic and diagnostic implications of Hsp90 activation. Trends Mol Med 2004, 10:283-290

10. Ali MM, Roe S, Vaughan CK, Meyer P, Panaretou B, Piper PW, Prodromou C, Pearl LH: Crystal structure of an Hsp90-nucleotide-p23/Sba1 closed chaperone complex. Nature 2006, 440:1013-1017.

11. Connell $P$, Ballinger CA, Jiang J, Wu Y, Thompson LJ, Höhfeld J, Patterson C: The co-chaperone CHIP regulates protein triage decisions mediated by heat-shock proteins. Nat Cell Biol 2001, 3:93-96.

12. Park MA, Zhang G, Mitchell C, Rahmani M, Hamed H, Hagan MP, Yacoub A, Curiel DT, Fisher PB, Grant S, Dent P: Mitogen-activated protein kinase kinase 1/2 inhibitors and 17-allylamino-17-demethoxygeldanamycin synergize to kill human gastrointestinal tumor cells in vitro via suppression of c-FLIP-s levels and activation of CD95. Mol Cancer Ther 2008, 7:2633-2648

13. Meacham GC, Patterson C, Zhang W, Younger JM, Cyr DM: The Hsc70 cochaperone CHIP targets immature CFTR for proteasomal degradation. Nat Cell Biol 2001, 3:100-105.

14. Shiau AK, Harris SF, Southworth DR, Agard D: Structural analysis of E. coli hsp90 reveals dramatic nucleotide-dependent conformational rearrangements. Cell 2006, 127:329-340.

15. Xu W, Marcu M, Yuan X, Mimnaugh E, Patterson C, Neckers L: Chaperonedependent E3 ubiquitin ligase CHIP mediates a degradative pathway for c-ErbB2/Neu. Proc Natl Acad Sci USA 2002, 99:12847-12852.

16. Liu X, Yue P, Schönthal AH, Khuri FR, Sun SY: Cellular FLICE-inhibitory protein down-regulation contributes to celecoxib-induced apoptosis in human lung cancer cells. Cancer Res 2006, 66:11115-11119.

17. Yerbes R, López-Rivas A: Itch/AIP4-independent proteasomal degradation of cFLIP induced by the histone deacetylase inhibitor SAHA sensitizes breast tumour cells to TRAIL. Invest New Drugs 2012, 30:541-547.

18. Chen S, Cao W, Yue P, Hao C, Khuri FR, Sun SY: Celecoxib promotes c-FLIP degradation through Akt-independent inhibition of GSK3. Cancer Res 2011, 71:6270-6281.

19. Chen S, Liu X, Yue P, Schönthal AH, Khuri FR, Sun SY: CCAAT/enhancer binding protein homologous protein-dependent death receptor 5 induction and ubiquitin/proteasome-mediated cellular FLICE-inhibitory protein down-regulation contribute to enhancement of tumor necrosis factor-related apoptosis-inducing ligand-induced apoptosis by dimethylcelecoxib in human non small-cell lung cancer cells. Mol Pharmacol 2007, 72:1269-1279.

20. Sun SY, Yue P, Dawson MI, Shroot B, Michel S, Lamph WW, Heyman RA, Teng M, Chandraratna RA, Shudo K, Hong WK, Lotan R: Differential effects of synthetic nuclear retinoid receptor-selective retinoids on the growth of human non small cell lung carcinoma cells. Cancer Res 1997 57:4931-4939

21. Liu X, Yue P, Zhou Z, Khuri FR, Sun SY: Death receptor regulation and celecoxib-induced apoptosis in human lung cancer cells. J Natl Cancer Inst 2004, 96:1769-1780.

22. Su L, Liu G, Hao X, Zhong N, Zhong D, Liu X, Singhal S: Death Receptor 5 and cellular FLICE-inhibitory protein regulate pemetrexed-induced apoptosis in human lung cancer cells. Eur J Cancer 2011, 47:2471-2478.

23. Chatterjee $M$, Jain $S$, Stühmer T, Andrulis $M$, Ungethüm U, Kuban RJ, Lorentz H, Bommert K, Topp M, Krämer D, Müller-Hermelink HK, Einsele H, Greiner A, Bargou RC: STAT3 and MAPK signaling maintain overexpression of heat shock proteins 90alpha and beta in multiple myeloma cells, which critically contribute to tumor-cell survival. Blood 2007, 109:720-728.

doi:10.1186/1475-2867-12-54

Cite this article as: Wang et al:: Down-regulation of cellular FLICEinhibitory protein (Long Form) contributes to apoptosis induced by Hsp90 inhibition in human lung cancer cells. Cancer Cell International 2012 12:54. 